\title{
An Efficient Localization Based on Directional Antenna for Wireless Sensor Networks (WSN's)
}

\author{
Dileep Kumar, Dr. Shirshu Varma, Member, IACSIT, IEEE
}

\begin{abstract}
In this paper we have introduced an algorithm for localization in wireless sensor network (WSN) with the help of directional antenna. Location of each node in a wireless sensor network play an important role after tracing or event detection on a particular node. Manually configuration of location about each node in wireless sensor environment is not feasible at the time of deployment. Our localization algorithm is based on the directional antenna, every node in sensor network is configured with directional antenna and few sensor nodes have GPS receiver are called anchor node. Anchor node helps to other normal nodes to localize them. Software has been developed for localization using $C$ language which will works in two phases: first one Query the sensor network and collect information about neighbours, distance and angle. Give this information as input to the software and the location of every node will be calculated. And second one is, compile the software for sensor nodes. Install the software in sensor nodes and the sensor nodes will calculate their location.
\end{abstract}

\section{INTRODUCTION}

Wireless sensor networks have become very popular for different application in present life. These are used for monitoring and control like, habitat monitoring, target tracking, disaster management, climate control etc. A wireless sensor node can be built easily using small electronic cheap technology. Wireless sensor networks (WSN) hold a promise to "dwarf previous revolutions in the information revolution" [1]. WSN are envisioned to consist of hundreds to thousands of sensor nodes communicating over a wireless channel, performing distributed sensing and collaborative data processing tasks for a variety of vital military and civilian applications [2]. Wireless sensor networks are densely deployed in sensing fields. They have very limited communication range and sensing range. Sensor node has very limited battery power and they are prone to failure. Wireless sensor nodes can be deployed in such a location where human being is impossible to reach physically. So, if a sensor node loses its battery power is becomes useless, hence the topology of wireless sensor networks changes frequently. In the wireless sensor networks localization is very important. If WSN is not able to provide location information about the event then tracking or event detection functions are not particularly useful. Sensor aware of their location can also improve routing efficiency by selective flooding or selective forwarding data only in the direction of the destination.

Manuscript received May 27, 2009

Dileep Kumar is with Department of Information Technology at Indian Institute of Information Technology, Allahabad, India. 211 011. (Phone: +91-9236741052, e-mail- iis2006007@ @iiita.ac.in )

Dr. Shirshu Varma is with Department of Information Technology at Indian Institute of Information Technology, Allahabad, India. 211011 (e-mail-shirshu@iiita.ac.in )
So localization is necessary in WSN. But problem is how a wireless sensor node can get the knowledge about its location.

Manually configuration of the location information in each node is not feasible at the time of deployment because the number of wireless sensor node is very large. The deployment of sensors can be done by throwing them in the sensing field by airplane, so sometimes it is not possible to go in the sensing field. Similarly we can not use GPS receiver in each node because it increases the cost of the node. Techniques based on GPS receiver are not useful for indoor applications. Many algorithms have been proposed earlier, each has its own benefits and limitations. A major shortcoming of all the algorithms is accuracy. No single algorithm can provide $100 \%$ accuracy. Properties of a good algorithm included Accuracy, Scalability and Minimum complexity.

\section{Localization in Wireless Sensor Networks}

\section{A. Concise explanation of Localization}

The process of finding location of the nodes is called localization. In this process we try to find out the approximate location of the sensor node with high accuracy. The process of localization collects information about the longitude, latitude and altitude of the sensor node. Localization can be classified in two ways: first one is Local/Relative localization in which location of a point is given with respect to a local point i.e. 4 meter from the pole. In this technique local reference points are used to describe location of an object. A reference frame is used for localization, and another one is Global/Absolute localization in which the location is given with respect to the world with the help of latitude, altitude, and longitude. Location can be specified in two ways: Geometric: the location of an object is specified using distance and angle. Topological: location is given by connection with landmarks.

\section{B. Why Localization?}

If we get some information from the sensor network and we don't know the location from where information is being generated, this information is useless. If we got information from the sensor network that there is fire, the next question will be "where". If we know the location information we can make arrangements for such accidental cases in time at that particular location within time. Most of routing protocols use flooding technique for routing of packets. If we know the location of each and every sensor node, a selective forwarding routing can be used to route packets. Selective forwarding reduces the network load and increases the network lifetime by saving power of individual node. Sensor data must be registered to its physical location 
to permit deployment of energy efficient routing schemes, source localization algorithms, and distributed compression techniques [3].

\section{Problems in Wireless Sensor Localization}

Most of the Wireless Networks uses GPS receiver for localization. But, the sensor network is very dense in nodes and using GPS receiver is not economically good. So GPS receiver can not be fitted in each and every sensor node because it increased the cost of sensor network and also it increase size of nodes. GPS receiver based techniques can not be used for indoor localization. GPS receiver techniques require satellite communication, so it needs more battery power. So finally GPS receiver based techniques are not suitable for sensor networks economically.

\section{Solution for Sensor Network Localization}

Whenever we are looking for localization in wireless sensor network, there could be two types of techniques used for sensor localization namely, Non-cooperative and Cooperative. In first one approach few base stations are used for localization where every sensor node receives signals directly from base station in which distance between node and base station can be measured on the basis of signal strength and time of arrival (TOI), and another approach a small no of anchor nodes are used in network, these anchor nodes have prior knowledge of location. An anchor node receives its location information either from GPS or from other means. These anchor nodes help to other normal nodes to localize themselves. In this approach there is no need to get signal from base station directly to localize a sensor node.

\section{E. Need of Independent Algorithm for Localization}

Sensor network needs an independent localization algorism because it is not possible to provide location information at the time of deployment. Some sensor networks have moving sensors, their location changes with respect to time. When a sensor node moves from one point to another point it needs localization. Sensors are deployed randomly in the sensing field. Location prediction of individual node is not possible at the time of deployment. New sensor nodes are also added to already deploy sensor network. So newly deployed sensor nodes need localization. Sensor nodes are usually node designed specifically to do localization. So sensor network needs individual localization algorithm.

\section{BACKGROUND AND LITERATURE WORK}

For localization in wireless sensor networks, a large no. of algorithms has been developed by many researchers but no one gives accurate location information. Some of the algorithms are based on mobile bacons where a single beacon (anchor) node is used [4]. The beacon node is connected to GPS receiver to find out its location. Beacon node is allowed to move in the WSN field such that each WSN node can get signal from at least three non linear positions. Beacon node can be positioned on a truck or on a airplane to move in the sensing field. Only a single beacon node is sufficient for localization. The proposed scheme is radio-frequency based, and thus no extra hardware is necessary.

In few systems signal strength is used for localization [5].
In which approach at least three anchor nodes are required in the range of unknown node. The unknown node constructs the rings on the basis of signal strength and calculates intersection area of rings. On the basis of gravity of intersection area unknown node estimates its location. Overall estimation depends on ring formulation. Radio propagation is usually not homogeneous. So there may be some errors. Handling of radio irregularity is also mentioned in the paper. Paper has assumed that more than half of the rings generated by RSSI comparison are correct. Again in next generation localized based sensor networks, multidimensional scaling and proximity distance map has been used [6]. In which multidimensional scaling and proximity distance mapping techniques are combined. $20 \%$ nodes are used as anchor nodes. The algorithm works in two phases, first it selects secondary nodes which are localized through multidimensional scaling. In the second phase unknown nodes are localized through proximity distance mapping.

Some of the systems use time of arrival (TOI) and time difference of arrival [7]. The system proposed by Frampton, in which author has been used time difference of arrival (TDOA) of acoustic signals where several sound sources of known location transmits the sound signals. Each sensor node receives this signal and record the time of arrival of the signal. Time difference of arrival is use for calculation of distance between anchor node and sensor nodes. Data from each sensor node is transmitted to a central processing unit. Using the distance between sensor node and different anchor nodes the location is calculated. Simulation and practical result has been shown. Simulation result proved that a sensor node can be localized with average error of $20 \mathrm{~cm}$. it has been also proved that increasing the number of anchor node the error in localization decreases and increasing the sensor nodes error increases.

Fading and mobility is also used by researchers. In those systems an approach based on mobility and fading has been used for finding localization [8].This technique uses only two anchor nodes. Anchor nodes are placed on the corner of the rectangular sensor field. Both the anchor nodes are placed on the same side of the rectangle. Distance between sensor and anchor nodes is measured on the basis of the signal strength. The constant power is transmitted from anchor nodes. Sensor nodes receive power and calculate distance by comparing transmitted power and received power.

Single Anchor node based localization in Sensor Network [9]. Has been developed by the Khan, H.M. Olariu, S. Eltoweissy, M. in this work the author has used a single anchor node fitted with directional antenna around its periphery. The number of directional antenna determines the accuracy and cost of the system. Anchor node is equipped with GPS receiver to find its location. Each sensor node is equipped with a transceiver. At any given time a sensor node either transmits or receive, but not both. All nodes have a fixed transmitter power. Coverage area of each antenna in the anchor node is determined by its beam width. With the help of directional antenna the angle between reference line and line joining the sensor node and anchor node is determined. The distance between sensor node and anchor node is determined using signal strength measurement. Once the 
distance and angle is determined the location can be determined using simple trigonometry formulae. The accuracy of the system depends on the beam width of the directional antenna. If two sensor nodes are at the same distance from the anchor node and in the range of the same antenna the location of both the nodes will be same. Accuracy of the system increases as the beam width of the directional antenna decreases.

\section{PROPOSED SOlution AND DETAIL OF AlgORITHM}

To localize node in a sensor network is a toughest problem. No localization algorithm can give the accurate location information of the sensor node. We can not use additional hardware the sensor nodes due to cost and size of sensor node. The localization algorithm should be designed to localize the sensor network. Our work is to find out an efficient localization algorithm for wireless sensor network. The idea is to use anchor nodes for localization. Anchor nodes are special nodes that know their physical location. Anchor nodes are connected to GPS to find out their location. Wireless sensor nodes that do not know their location are known as unknown nodes. Unknown nodes communicate with anchor nodes. Here in this work we are using directional antenna to for localization. The directional antenna is used to calculate angle of arrival, on the basis of signal strength. The distance between anchor node and unknown node is calculated. After getting distance and angle information mathematical calculations are done to calculate the location of individual nodes. Our main motive is to reduce complexity of algorithm and error in distance measurement. This algorithm basically uses angle of arrival (AOA).

\section{A. Angle of Arrival (AOA)}

In the localization process angle of arrival is also used. Angle of arrival is used in the contribution of RSS. If there is a base station from which a sensor node is receiving signal, distance is calculated using RSS and if we can calculate the angle of the line joining the sensor node and base station and a reference line then we can calculate location of sensor node. Angle of arrival is a technique to determine the direction of signal incident on an antenna array. AOA use time difference of arrival (TDOA) for measuring direction of signal. Phase difference of different signals incident on different element of antenna array is used to calculate direction. It is just like reverse beam forming. Figure (1) shows the angle of arrival.

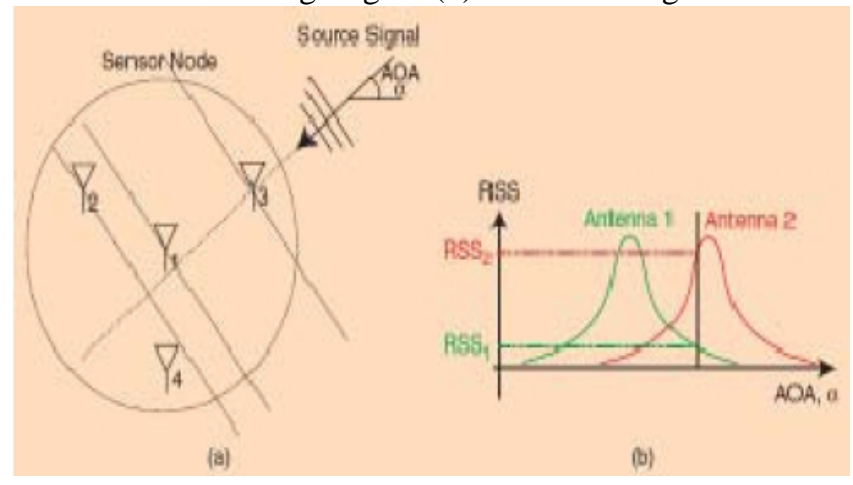

Fig. 1. Angle of Arrival

Lets the angle of the line with reference line is $\theta$ and the distance between sensor node and base station is $\mathrm{d}$ having location $(x, y)$ then, Location of the sensor node is calculated as follows,

$$
\begin{aligned}
& \mathrm{X}^{\prime}=\mathrm{d} \cos \theta \\
& \text { And } \\
& \mathrm{Y}^{\prime}=\mathrm{d} \sin \theta .
\end{aligned}
$$

Angle of arrival can be calculated using directional antenna.

$$
\mathrm{y}
$$

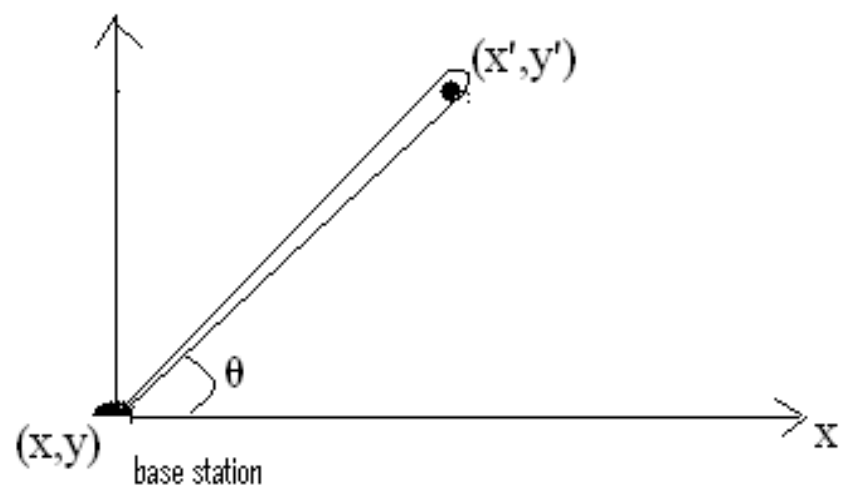

Fig. 2. Rotational Directional Antenna

A rotating directional antenna can be used for localization. The rotation directional antenna as shown in figure (2) rotates at a constant speed. Every sensor node receive signal from the directional antenna. Different sensor nodes at different location receives signal at different time. This time difference is used to decide angle. Directional antenna array can be used to cover whole area. If the beam width of directional antenna is ǿ, there is 360/ǿ directional antenna needed to cover whole area.

\section{B. Algorithmic Implementation}

The complete algorithm comprises of five steps. In the first step nodes are randomly deployed. In second step the nodes starts to find out their neighbours. In the third step their local information is exchanged about their location $(\mathrm{x}, \mathrm{y})$ and the distance. In fourth step actual localization is done. The final and fifth step calculated localization where location is adjusted by applying rotation and scaling.

\section{B.1. Deployment}

In this phase the nodes are uniformly deployed in the sensing field. Here in simulation we have used a uniform () function to generate $(\mathrm{x}, \mathrm{y})$ coordinates of the sensor node. Uniform () is a function which generates uniformly distributed numbers between two predefined numbers with equal probability.

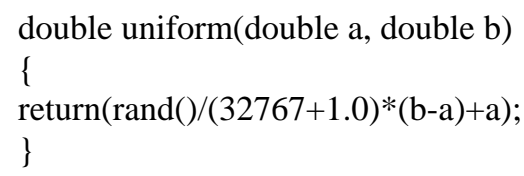

This function returns a random number between $a$ and $b$ with equal probability. Figure (3) shows the deployment of nodes in sensor network. 


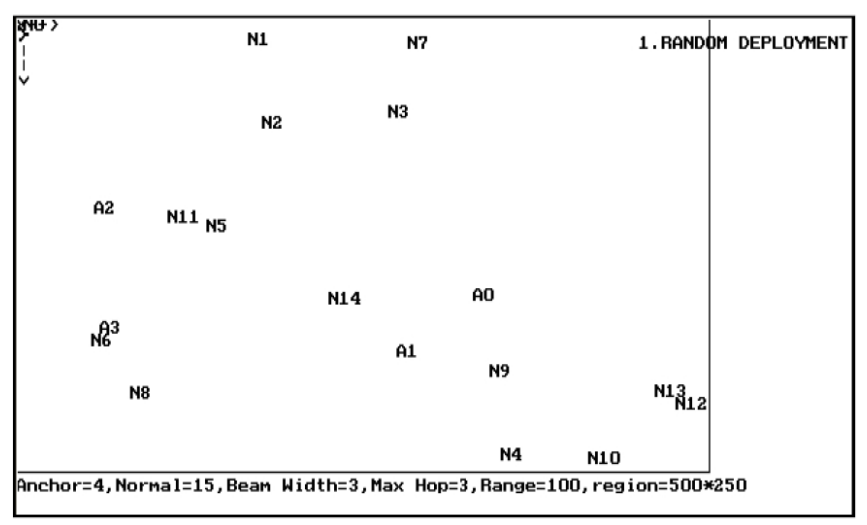

Fig. (3). Random Deployment

\section{B. 2. Finding Neighbours}

A sensor node is called neighbour of another node if they both are in the communication range of one another. After deployment each node starts to find its neighbour. Each node will have list of its neighbour. There is a predefined communication range. Here in our simulation we have used different communication ranges particularly for the results shown in figure (4) communication range is $100 \mathrm{~m}$. Two nodes are neighbours if the distance between two nodes is less or equal to predefined range. Here in our simulation we have used actual coordinates of node to calculate distance between two nodes. The function used to calculate distance is

double distance(double $\mathrm{x} 1$, double $\mathrm{y} 1$, double $\mathrm{x}$, double y2)

$$
\{
$$

return(double $(\operatorname{sqrt}(((x 1-x 2) *(x 1-x 2))+((y 1-y 2) *(y 1-y 2)))))$;

$$
\text { \} }
$$

Where (x 1, y2) coordinates of first node and (x 2, y2) are coordinates of another node. Angle between two nodes is also calculated. The function used to calculated angle of line joining two nodes with respect to $\mathrm{x}$-axis is

double angle(double $\mathrm{x} 1$, double $\mathrm{y} 1$, double $\mathrm{x} 2$, double $\mathrm{y} 2$ ) \{

return(atan2((y2-y1),(x2-x1)));

\}

Figure (4) illustrates the finding neighbours in WSN

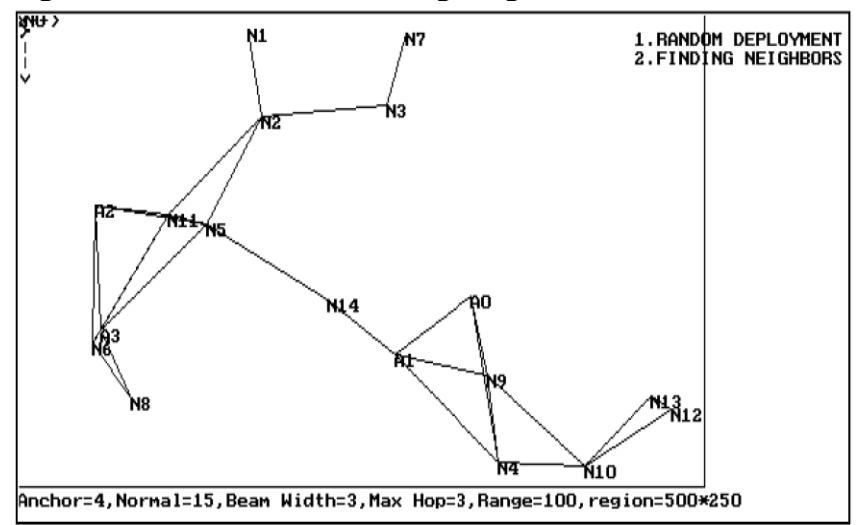

Fig. (4) Finding Neighbours

\section{B. 3. Information Exchange}

In this algorithm anchor nodes starts information exchange. When a node gets information from its neighbor, it will localize itself and sends its location information to its neighbours so that they can localize themselves. Also sent information contains estimated location of itself, transmitted power and the antenna id from which information is being sent. Transmitted power is used to calculate distance between nodes. Antenna id is used to calculate angle of the connecting line with respect to $\mathrm{x}$-axis. If a node has received information from a neighbor it won't send information to that neighbor. Figure (5) shows the information exchanging between nodes.

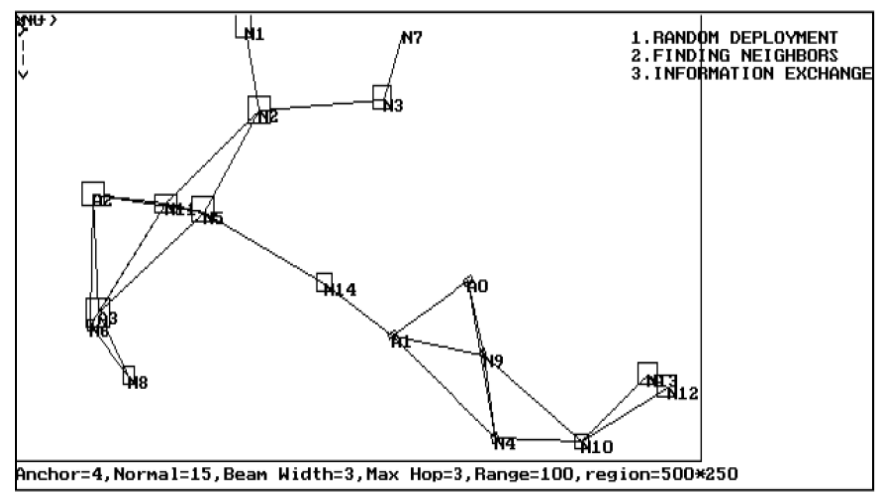

\section{B. 3. Localization}

Fig. (5) Information Exchange

Whenever a node receives information from its neighbor it will calculate its location. When a node receive information from an anchor node it will use (x, y) coordinates of anchor node, distance from the anchor nodes and angle with anchor node to estimate its location. If the beam width of the directional antenna is $\Theta$ there is always an uncertainty of \pm $\theta / 2$. If the error in distance is $\mathrm{e} \%$ and distance is $\mathrm{d}$ then there is always an uncertainty of $\pm \mathrm{d} \times \mathrm{e} / 100$. The node calculates its location area with given error in angle and distance. After calculating location area the complete area is transmitted to its neighbor so that they can estimate their location. When a node gets information from more than two neighbors the grid scan algorithm is applied to calculate overlap area. Figure (6) shows the localization in wireless sensor network.

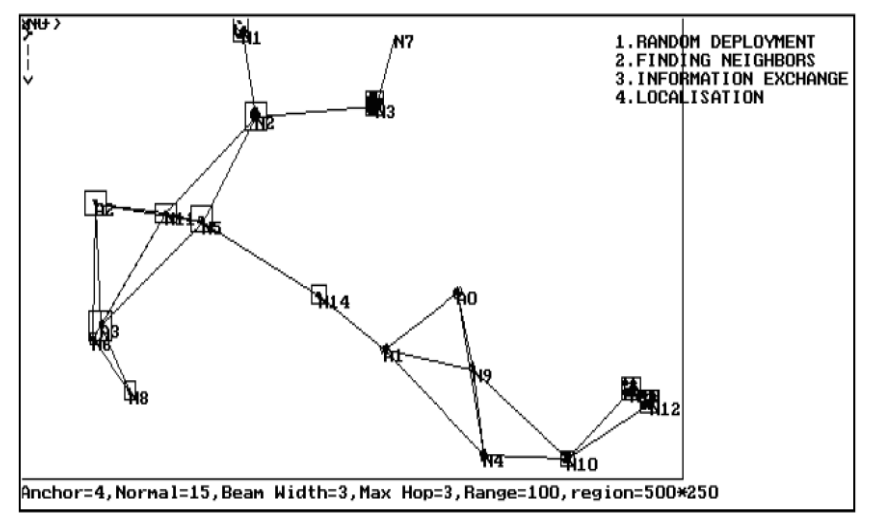

Fig. (6) Localization

\section{B. 4. Adjustment and Scaling}

When localization is applied in the network the anchor nodes also estimates their location with respect to other anchor nodes. So we know the actual location and estimated location of each anchor nodes. We calculate error in the actual location and estimated location. This error is applied to 
the unknown nodes to adjust their location. Figure (7) shows the adjustment and scaling between nodes.

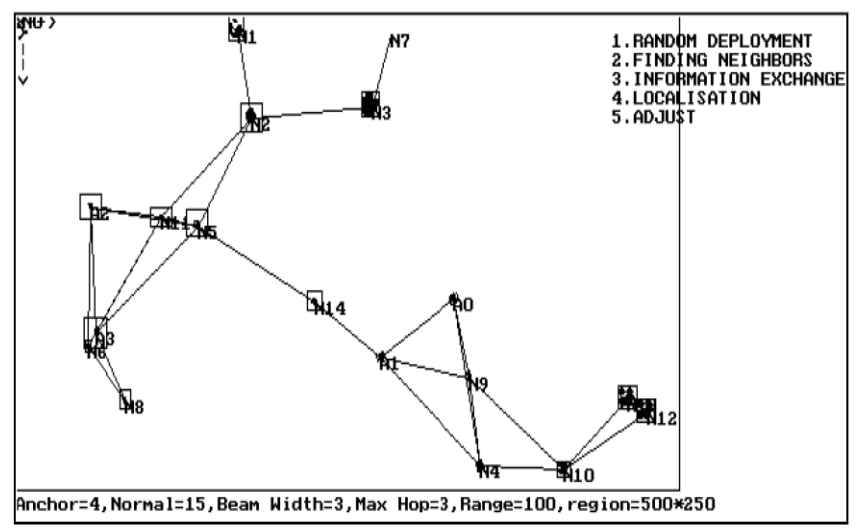

Fig. (7) Adjustment and Scaling

\section{Simulation and Results}

The simulation for this algorithm has been done using $\mathrm{C}$ language. We assume that all the data available at the time of simulation. Nodes are randomly deployed in the area. We have simulated the work with different number of anchor nodes and normal sensor nodes. Every time we have used $10-30 \%$ anchor nodes and taken the simulation results. Simulation results shows that if we increase number of anchor nodes we get better accuracy. We have used 6\% error in the distance and beam width is 30 . Every time a region is calculated for every sensor node. The average of the region is calculated for final location. The input for the simulation is as follows for $500 \times 200 \mathrm{~m} 2$ region:

- 50 to 200 normal nodes.

- b. $10 \%$ to $30 \%$ anchor nodes.

- Error in distance measurement is $6 \%$.

- Beam width of directional antenna is 30 .

- Random deployment.

- Nodes are stationary.

- Maximum hop to flow information from the anchor node is 3 to 8 .

We have applied this algorithm on different number of nodes in WSN, here are the results for 15 nodes and we got the information about location as shows in table (1).

TABLE (1). LOCALIZATION RESULT WITH 15 NORMAL NODES
Here (Tx, Ty) is the true location of the normal node. $(\mathrm{Cx}, \mathrm{Cy})$ is calculated location of the sensor nodes. Here we see that the normal nodes $0,1,2,3,7,12$ and 13 do not have location information. If a node can not get information from the anchor node it can not be localized. Error in localization is calculated as shown in table (2).

TABLE (2). ERROR IN LOCALIZATION

\begin{tabular}{|c|c|c|c|c|c|c|c|c|c|}
\hline TxbarA & TybarA & CxbarA & CybarA & TxbarN & TybarI & CxbarN & CybarN & error $X$ & error Y \\
\hline 90 & 119 & 150 & 131 & 118 & 104 & 105 & 139 & 1 & 0.9 \\
\hline \multicolumn{2}{|c|}{ Node } & \multicolumn{2}{|l|}{$\mathrm{Tx}$} & \multicolumn{2}{|l|}{$\mathrm{Ty}$} & \multicolumn{2}{|c|}{$\mathrm{Cx}$} & \multicolumn{2}{|l|}{$\mathrm{Cy}$} \\
\hline \multicolumn{2}{|c|}{ No } & \multicolumn{2}{|l|}{2} & \multicolumn{2}{|l|}{0} & & & \\
\hline \multicolumn{2}{|l|}{ N1 } & \multicolumn{2}{|l|}{83} & \multicolumn{2}{|l|}{6} & & & & \\
\hline \multicolumn{2}{|c|}{ N2 } & \multicolumn{2}{|l|}{88} & \multicolumn{2}{|l|}{53} & & & & \\
\hline \multicolumn{2}{|c|}{ N3 } & \multicolumn{2}{|c|}{132} & \multicolumn{2}{|l|}{39} & & & & \\
\hline \multicolumn{2}{|c|}{ N4 } & \multicolumn{2}{|c|}{175} & \multicolumn{2}{|l|}{189} & \multicolumn{2}{|l|}{174} & \multicolumn{2}{|l|}{188} \\
\hline \multicolumn{2}{|l|}{ N5 } & \multicolumn{2}{|l|}{68} & \multicolumn{2}{|l|}{88} & \multicolumn{2}{|c|}{66} & \multicolumn{2}{|l|}{87} \\
\hline \multicolumn{2}{|l|}{ N6 } & \multicolumn{2}{|l|}{27} & \multicolumn{2}{|l|}{139} & \multicolumn{2}{|c|}{26} & \multicolumn{2}{|l|}{139} \\
\hline \multicolumn{2}{|c|}{ N7 } & \multicolumn{2}{|c|}{141} & 8 & & & & & \\
\hline N8 & & 41 & & 163 & & 40 & & 162 & \\
\hline N9 & & 171 & & 152 & & 171 & & 151 & \\
\hline N1 & & 206 & & 191 & & 205 & & 189 & \\
\hline N1 & & 54 & & 85 & & 53 & & 84 & \\
\hline N1 & & 238 & & 167 & & & & & \\
\hline N1 & & 230 & & 162 & & & & & \\
\hline N1 & & 112 & & 120 & & 111 & & 119 & \\
\hline
\end{tabular}

Where,

TxbarA =average of true $\mathrm{x}$ coordinate of all the anchor nodes TybarA =average of true $y$ coordinate of all the anchor nodes CxbarA $=$ average of calculated $\mathrm{x}$ coordinate of all the anchor nodes

CxbarA =average of calculated y coordinate of all the anchor nodes.

The same algorithm has applied on 10 anchor nodes, 80 normal nodes, communication range $25 \mathrm{~m}$ and $\max$ hop 5 within the range of $200 * 200$ regions, result for deployment

(8) and table (3) respectively.

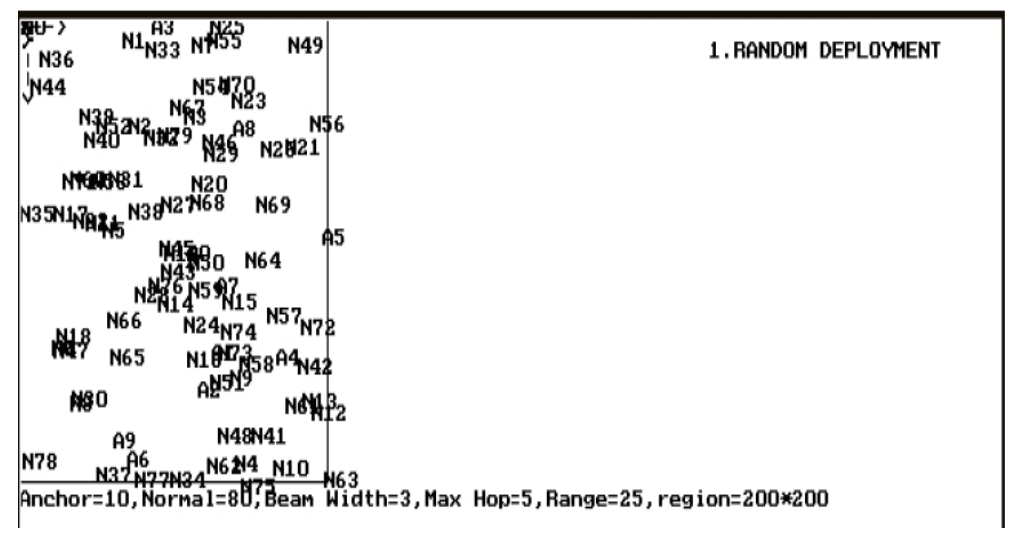

Fig. (8). Random Deployment 
TABLE (3). ERROR IN LOCALIZATION FOR 80 NORMAL AND 10 ANCHOR NODES

\begin{tabular}{|r|r|r|r|r|r|r|r|r|r|}
\hline TxbarA & TybarA & CxbarA & CybarA & TxbarN & TybarN & Adxbar & Adybar & $\begin{array}{l}\text { Error } \\
\text { X }\end{array}$ & $\begin{array}{l}\text { Error } \\
\text { Y }\end{array}$ \\
\hline 124 & 93 & 120 & 89 & 98 & 101 & 96 & 102 & 2 & 1 \\
\hline
\end{tabular}

A mean squire error with respect to number of anchor nodes, beam width and maximum hop is calculated using the formula

$$
\text { mean square error }=\sqrt{\frac{1}{N} \sum_{1}^{N}(T x-C x)^{2}+\frac{1}{N} \sum_{1}^{N}(T y-C y)^{2}}
$$

Where $\mathrm{N}$ represents the number of anchor nodes, (Tx, Ty) are the true location of normal nodes and $(\mathrm{Cx}, \mathrm{Cy})$ are the calculated location of the normal nodes. Graphical representation of number of anchor nodes, beam width and maximum hop versus mean squire error is shown in figure $(9$, 10 and 11) respectively. Here we have taken number of anchor nodes 20 , number of hops 3 and the beam width is varied from 3 degree to 30 degree.

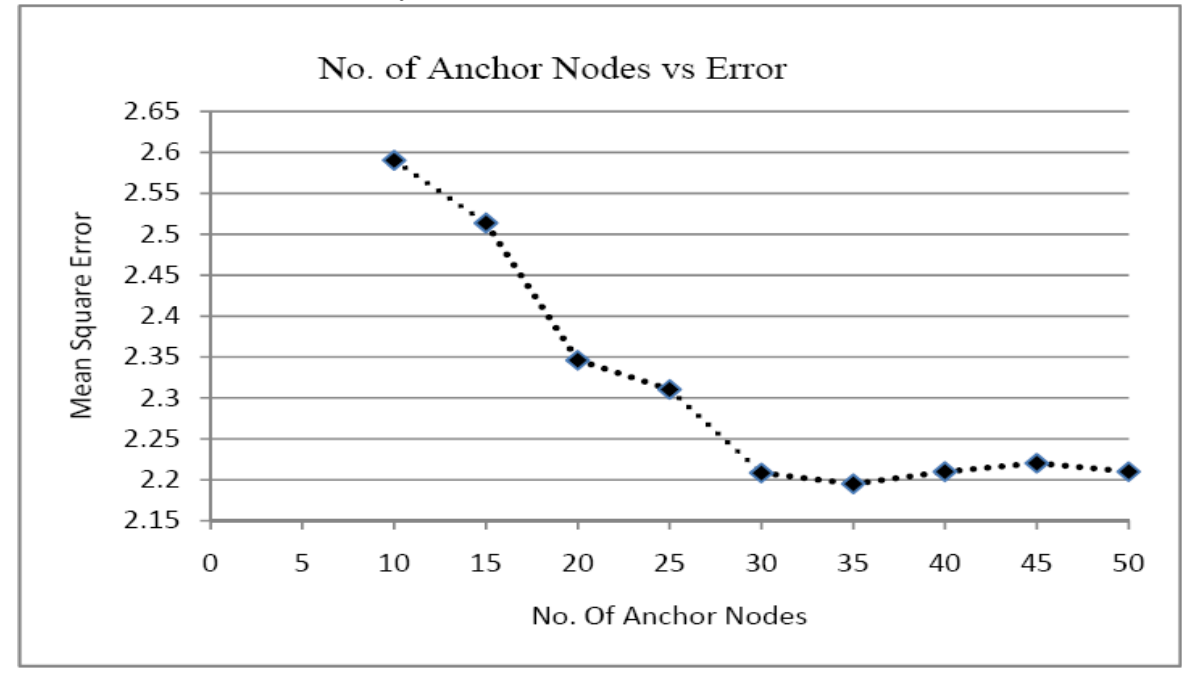

Figure (9). Anchor nodes vs Mean squire error

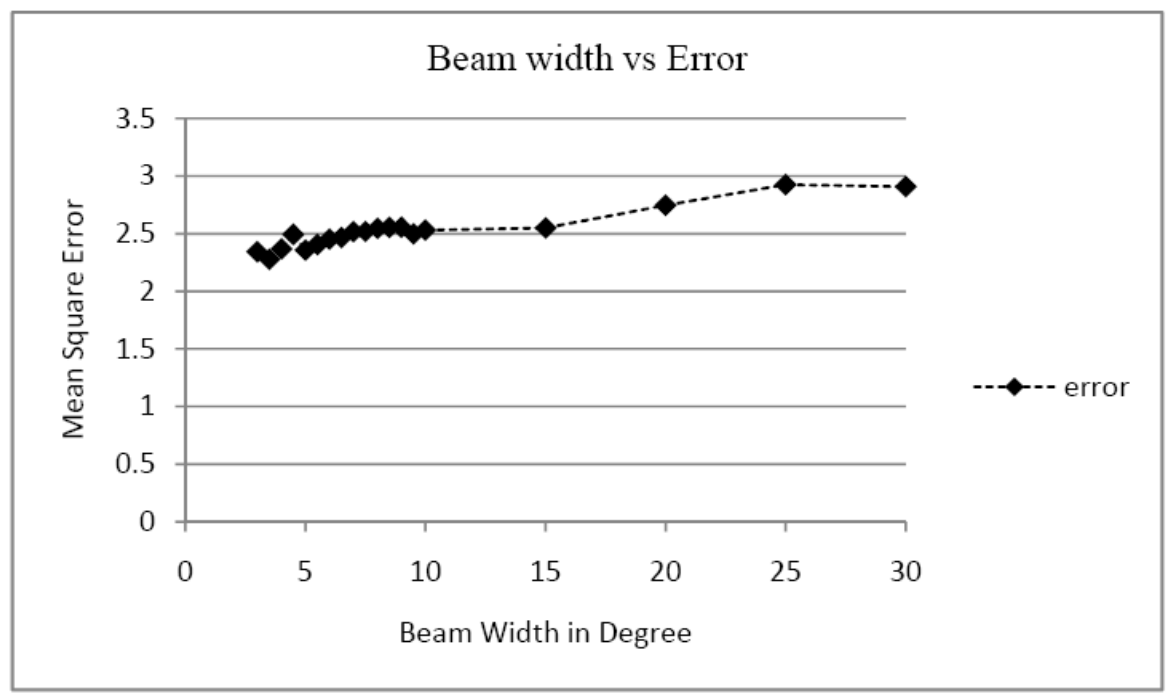

Figure (10). Beam width vs Mean squire error 


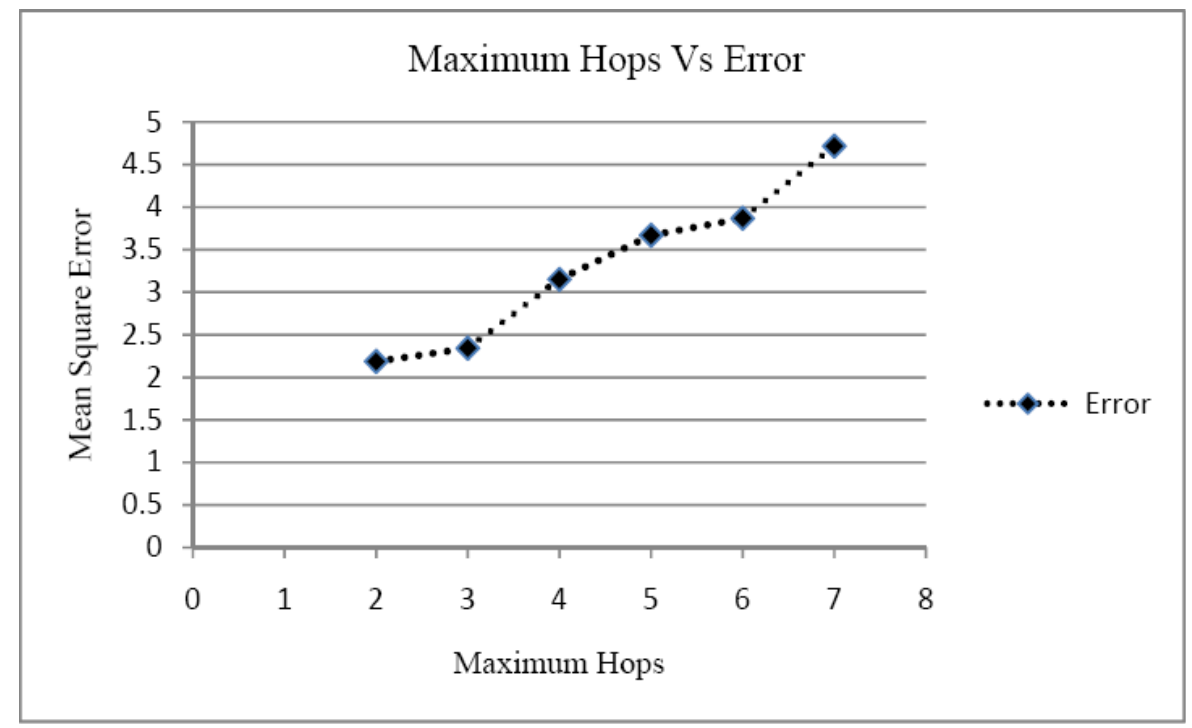

Figure (11). Max hopes vs Mean squire error

\section{A. Result Summary}

The proposed localization algorithm works fine and provides better accuracy; in this approach if the number of anchor nodes increases then the accuracy in localization also increases. Accuracy also increases if the number of anchor neighboring nodes increases. If we increase the number of hop for information flow the accuracy of the information decreases, but the number of the neighboring nodes increases, so finally the accuracy of localization increases. We have used different number of nodes, hop, and beam width and communication range to check the accuracy of the algorithm. Using different result it is proven that this algorithm gives the better accuracy.

\section{CONCLUSION AND FUTURE WORK}

In this work a localization algorithm is suggested using directional antenna. It is easy to find out the neighbors of any node. If a node can receive signals from the any other node these nodes are called neighbor. The distance between two neighboring node can be easily calculated using RSS technique. After calculating the angle of arrival, distance a normal node starts to localize itself. A node calculated its approximate area. If a node gets signal from more than 2 nodes it calculates its overlap area. Calculation of overlap area is done by grid scan algorithm. The suggested algorithm executed on the individual computer so there is no memory limitation and processing limitation. Only a little information exchange is done in the sensor network. There is no battery loss. Only thing is that directional antenna. The accuracy of the algorithm increases as the beam width decreases.

We have implemented this work for stationary sensor nodes; in future this algorithm can be implemented for the mobile sensor nodes.
The authors express their sincere gratefulness to Dr. M. D. Tiwari, Director IIIT, Allahabad, for his encouragement and support through out the course of this work and also for facilitating technical and literature facilities, required in the development of this work.

\section{REFERENCES}

[1] Costa, J.A.; Patwari, N.; Hero, A.O., III Acoustics, Speech, and Signal Processing, 2005. Proceedings.(ICASSP apos;05). IEEE International Conference on "Achieving High-Accuracy Distributed Localization in Sensor Network"

[2] Aram Galstyan', Bhaskar Krishnamachari" Kristina Lermanl and Sundeep "Distributed Online Localization in Sensor Networks Using a Moving Target" Department of Electrical Engineering- Systems University of Southern California.

[3] I. Akyildiz, W. Su, Y. Sankarasubramaniam, and E. Cayirci, "A survey on sensor networks," IEEE Communication Magazine, vol. 40, pp. 102-116, Aug. 2002.

[4] Sichitiu, M.L.; Ramadurai, V.; "Localization of wireless sensor networks with a mobile beacon", Mobile Ad-hoc and Sensor Systems, 2004 IEEE International Conference on 25-27 Oct. 2004.

[5] Chong Liu; Kui Wu; Tian He; "Sensor localization with Ring Overlapping based on Comparison of Received Signal Strength Indicator" Mobile Ad-hoc and Sensor Systems, 2004 IEEE International Conference on 25-27 Oct. 2004.

[6] King-Yip Cheng, King-Shan Lui and Vincent Tam" Localization in Sensor Networks with Limited Number of Anchors and Clustered Placement”. 2007 IEEE International on Wireless Communications and Networking Conference, during 11-15 March 2007.

[7] Frampton, K.D.; "Acoustic self-localization in a distributed sensor network" Sensors Journal, IEEE Volume 6, Issue 1, Feb. 2006.

[8] Bergamo, P.; Mazzini, G.; Localization in sensor networks with fading and mobility Personal, Indoor and Mobile Radio Communications, 2002. The 13th IEEE International Symposium on Volume 2, 15- 18 Sept. 2002.

[9] Khan, H.M. Olariu, S. Eltoweissy, M. Efficient single-anchor localization in sensor networks Dependability and Security in Sensor Networks and Systems, 2006. DSSNS 2006. Second IEEE Workshop on Publication Date: 24-28 April 2006.

\section{ACKNOWLEDGEMENT}


Dileep Kumar received his B.Tech degree in Information Technology form BSA College of Engineering \& Technology, Mathura (India) in 2006. He has done M.Tech. in Information technology with specialization of Intelligent Systems from Indian Institute of Information technology, Allahabad (India) in 2008. Currently he is working as a lecturer in department of Information Technology in Institute of Engineering and Technology, Alwar-Rajasthan (India). His research and teaching interest included Wireless technology, Telecommunication, Wireless Sensor Network, Artificial Intelligent, Machine Vision and Soft Computing.
Email- iis2006007@iiita.ac.in

Dr. Shirshu Varma is currently an Assistant Professor and HOD of Department of Wireless Communication Engineering, Indian Institute of information Technology, Allahabad- 211 012, Uttar Pradesh (India). He has published over 35 papers in international journals as well as in international/national conferences. He has the specialization and an experience of 20 years in the area of communication Systems, Wireless Sensor Networks, mobile Computing, Digital Signal processing and telecommunication systems. Email- shirshu@iiita.ac.in 